\title{
Singular Temperature Dependence of the Equation of State of Superconductors with Spin-Orbit Interaction in Low-Temperature Region - II
}

\author{
Yu. N. Ovchinnikov ${ }^{1,2}$ \\ Received: 28 February 2018 / Accepted: 23 March 2018 / Published online: 26 April 2018 \\ (c) The Author(s) 2018
}

\begin{abstract}
We extend a previous study of the space and temperature dependence of the order parameter in an ultrathin superconducting film in a longitudinal magnetic field to the vicinity of the critical transition surface. In these films, the spin-orbit interaction can be sizeable. The calculations are restricted to the low-temperature regime. Special attention is given to the zero temperature limit where the state with the maximum value of the critical field is investigated for the given strength of the spin-orbit interaction.
\end{abstract}

Keywords Superconductivity $\cdot$ Inhomogeneous state $\cdot$ Spin-orbit interaction $\cdot$ Order parameter $\cdot$ Singularity in low temperature region

\section{Introduction}

Very thin film deposited on a substrate can be in a superconducting state $[1,2]$, even in very strong longitudinal magnetic fields and with strong spin-orbit interactions. In paper [1], single atomic layer films of $\mathrm{Pb}$ on $\mathrm{Si}(111)$ substrate have been studied. Superconducting transition temperature was $1.83 \mathrm{~K}$. The upper critical field was estimated to be $1450 \mathrm{G}$. The experimental data for order parameter $\Delta$ are in a good agreement with the BCS theory. In the paper [2], the superconductivity with Tc above $100 \mathrm{~K}$ in the FeSe on the substrate $\mathrm{SrTiO}_{3}$ was estimated. The temperature dependence of critical current $\mathrm{J}_{c}$ was fitted to the Ginzburg-Landau functional. It was found that $\mathrm{J}_{c}$ decreased with increasing magnetic field up to $11 \mathrm{~T}$ at $3 \mathrm{~K}$.

The order parameter $\Delta$ in such a system is the function of three parameters: magnetic field, spin-orbit interaction, temperature. Equation $\Delta=0$ creates surface in this space: critical transition surface. The critical transition surface on

Yu. N. Ovchinnikov

ovc@itp.ac.ru

1 Max-Planck-Institut für Physik komplexer Systeme, 01187 Dresden, Germany

2 Landau Institute for Theoretical Physics, Chernogolovka 142432, Moscow District, Russia the subspace spanned by magnetic field, spin-orbit interaction, and temperature was investigated in a low-temperature region in papers [3,4]. The point $T=0$ is a singular point for such a system. It is expected that near the transition surface the behavior of the order parameter also will be nontrivial and will attract special interest for experimental investigations. In this paper, we present the results of theoretical investigation of the space and temperature dependence of the order parameter in the vicinity of critical transition surface. Note that critical values of the longitudinal magnetic field and spin-orbit interaction in the inhomogeneous state are nearly twice as large as the same values are for homogeneous state. We investigate the state starting from the state with the maximal value of the critical magnetic field for fixed value of the spin-orbit interaction at $T=0$.

\section{Equation System for the Superconducting Order Parameter}

For a thin superconducting film, deposited on a substrate, the spin-orbit interaction can be taken in the form $[5,6]$

$\hat{V}_{\mathrm{SO}}=\frac{-i \hbar^{2}}{2 m^{2} c^{2}}\left(\left[\frac{\partial U}{\partial \mathbf{r}} \times \frac{\partial}{\partial \mathbf{r}}\right] \hat{\mathbf{S}}\right)$

where $\hat{\mathbf{S}}$ is the spin operator of an electron, $m$ is the electron mass, $c$ is the speed of light, and $U$ is the self-consistent 
potential. In normal metals, the Green's function is a $2 \times 2$ matrix and satisfied the equation

$\left(\frac{\partial}{\partial \tau}+\hat{L}\right) \hat{G}=\delta\left(\mathbf{r}-\mathbf{r}^{\prime}\right) \delta\left(\tau-\tau^{\prime}\right)$

where $\tau$ is imaginary time and the operator $\hat{L}$ is

$\hat{L}=-\frac{\hbar^{2}}{2 m} \frac{\partial^{2}}{\partial \mathbf{r}^{2}}+\hat{V}_{\mathrm{SO}}-\mu-\frac{e \hbar}{m c}(\mathbf{H S})$.

The last term is the Zeeman energy, $\mu$ is the chemical potential, and $\mathbf{H}$ is an in-plane external magnetic field. The eigenfunctions of operator $\hat{L}$ are of the form

$\left(\begin{array}{l}\psi_{1}^{(n)} \\ \psi_{2}^{(n)}\end{array}\right)=\exp \left(\frac{i p_{x}}{\hbar} x+\frac{i p_{y}}{\hbar} y\right) \chi_{n}(z)\left(\begin{array}{l}f_{1}^{(n)} \\ f_{2}^{(n)}\end{array}\right)$.

The $\chi_{n}(z)$ are normalized eigenfunctions of the operator

$\left(-\frac{\hbar^{2}}{2 m} \frac{\partial^{2}}{\partial z^{2}}+U(z)\right) \chi_{n}(z)=\epsilon_{n} \chi_{n}(z), \quad n=0,1,2, \ldots$

In the subspace with given values of $p_{x}, p_{y}$, and $n$, the vector $\left(f_{1}^{(n)}, f_{2}^{(n)}\right)$ is a solution of the equation

$$
\begin{aligned}
& {\left[-\frac{\hbar^{2}}{4 m^{2} c^{2}} \hat{\alpha}_{n}\left(\begin{array}{cc}
0 & p_{x}+i p_{y} \\
p_{x}-i p_{y} & 0
\end{array}\right)\right.} \\
& \left.-\left(\begin{array}{cc}
0 & h_{x}-i h_{y} \\
h_{x}+i h_{y} & 0
\end{array}\right)\right]\left(\begin{array}{l}
f_{1}^{(n)} \\
f_{2}^{(n)}
\end{array}\right)=\lambda^{(n)}\left(\begin{array}{l}
f_{1}^{(n)} \\
f_{2}^{(n)}
\end{array}\right) .
\end{aligned}
$$

with

$\hat{\alpha}_{n}=\int_{-\infty}^{\infty} d z \frac{\partial U}{\partial z}\left|\chi_{n}(z)\right|^{2}, h=\mu_{B} \mathbf{H}, \mu_{B}=\frac{e \hbar}{2 m c}$.

Eigenvalues $\lambda^{(n)}$ are

$\lambda_{ \pm}^{(n)}= \pm\left\{\frac{\left(\tilde{\alpha}_{n}\right)^{2}}{4} v_{F}^{2} p^{2}+h^{2}+\left(h_{x} p_{y}-h_{y} p_{x}\right) v_{F} \cdot \tilde{\alpha}_{n}\right\}^{\frac{1}{2}}$

where

$\tilde{\alpha}_{n}=\hat{\alpha}_{n} \frac{\hbar}{2 m^{2} c^{2} v_{F}}$.

and $v_{F}$ is Fermi velocity.

The eigenfunctions $f_{ \pm}^{(n)}$ are found to be

$f_{+}^{(n)}=\frac{1}{\sqrt{2}}\left[1 ;-\frac{1}{\lambda_{+}^{(n)}}\left(\frac{\tilde{\alpha} v_{F}}{2}\left(p_{y}-i p_{x}\right)+\left(h_{x}+i h_{y}\right)\right)\right]$

$f_{-}^{(n)}=\frac{1}{\sqrt{2}}\left[-\frac{1}{\lambda_{-}^{(n)}}\left(\frac{\tilde{\alpha} v_{F}}{2}\left(p_{y}+i p_{x}\right)+\left(h_{x}-i h_{y}\right)\right) ; 1\right]$.
In a normal metal, Green's function decomposes into four blocks (see also, consideration of normal metal without spin-orbit interaction [7])

$\left(\begin{array}{ccc}\hat{G}_{++}^{(0)} & ; & 0 \\ 0 & ; & \hat{G}_{--}^{(0)}\end{array}\right)$

and satisfies the equation

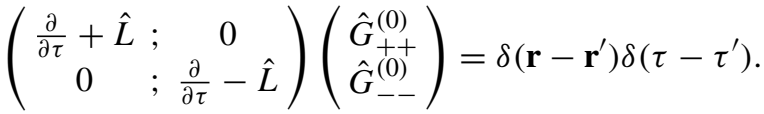

From (3), (8), (10), and (11), we obtain

$$
\begin{aligned}
\hat{G}_{++}^{(0)}= & T \sum_{\omega} \sum_{n} \chi_{n}(z) \chi_{n}^{*}\left(z^{\prime}\right) \\
& \times \sum_{\lambda_{+}^{(n)}} \int_{-\infty}^{\infty} \frac{d p_{x} d p_{y}}{(2 \pi \hbar)^{2}}\left(f(\mathbf{p})^{(n)}\left(f(\mathbf{p})^{(n)}\right)^{+}\right)_{+} \\
& \times \frac{\exp \left(\frac{i \mathbf{p}}{\hbar}\left(\mathbf{r}-\mathbf{r}^{\prime}\right)\right) \exp \left(-i \omega\left(\tau-\tau^{\prime}\right)\right)}{-i \omega+\frac{\mathbf{p}^{2}}{2 m}+\epsilon_{n}-\mu+\lambda_{+}^{(n)}(\mathbf{p})} \\
\hat{G}_{--}^{(0)}= & T \sum_{\omega} \sum_{n} \chi_{n}(z) \chi_{n}^{*}\left(z^{\prime}\right) \\
& \times \sum_{\lambda_{-}^{(n)}} \int_{-\infty}^{\infty} \frac{d p_{x} d p_{y}}{(2 \pi \hbar)^{2}}\left(f(\mathbf{p})^{(n)}\left(f(\mathbf{p})^{(n)}\right)^{+}\right)_{-} \\
& \times \frac{\exp \left(\frac{i \mathbf{p}}{\hbar}\left(\mathbf{r}-\mathbf{r}^{\prime}\right)\right) \exp \left(-i \omega\left(\tau-\tau^{\prime}\right)\right)}{-i \omega-\frac{\mathbf{p}^{2}}{2 m}-\epsilon_{n}+\mu-\lambda_{-}^{(n)}(\mathbf{p})} .
\end{aligned}
$$

In (12), quantity $\left.\left(f(\mathbf{p})^{(n)}(f(\mathbf{p}))^{(n)}\right)^{+}\right)_{ \pm}$is matrix $2 * 2$ with usual matrix product. In order to have a strong enough spinorbit interaction, the film thickness should be very small (i.e., of the order of interatomic distances). Then, the energy distance in $\epsilon_{n}$ is large. This circumstance enables us to keep only the term $n=0$ in the sum over $n$. The function (10) forms a complete basis in terms of which we can express the electron-electron interaction as

$H_{\mathrm{int}}=\frac{1}{2} \int d^{3} \mathbf{r} d^{3} \mathbf{r}^{\prime} V\left(\mathbf{r}-\mathbf{r}^{\prime}\right)\left(\psi_{\nu}^{+}(\mathbf{r})\left(\psi_{\mu}^{+}\left(\mathbf{r}^{\prime}\right) \psi_{\mu}\left(\mathbf{r}^{\prime}\right)\right) \psi_{\nu}(\mathbf{r})\right)$

with indexes $v, \mu= \pm$. We assume below that the potential $V$ is $\delta$-function like: $V\left(\mathbf{r}-\mathbf{r}^{\prime}\right)=V_{0} \delta\left(\mathbf{r}-\mathbf{r}^{\prime}\right)$. We consider Cooper pairing of the type $\left\langle\psi_{+}(\mathbf{r}) \psi_{-}\left(\mathbf{r}^{\prime}\right)\right\rangle-$ electrons only, i.e., Cooper pairs formed from different Fermi surfaces $[7,8]$. 
In superconductor, Green's function $\tilde{\hat{G}}$ is matrix $(4 \times 4)$ and satisfies the equation

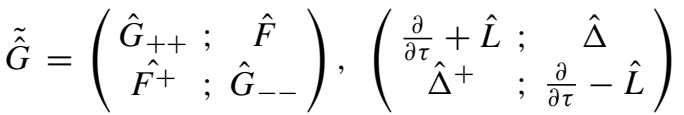

$$
\begin{aligned}
& \tilde{\hat{G}}=\delta\left(\mathbf{r}-\mathbf{r}^{\prime}\right) \delta\left(\tau-\tau^{\prime}\right)
\end{aligned}
$$

The operator $\hat{\Delta}(\mathbf{r})$ is [3]

$\hat{\Delta}(\mathbf{r})=\Delta(\mathbf{r})\left(\begin{array}{c}1 ; 0 \\ 0 ; 1\end{array}\right) T r$

where $\operatorname{Tr}$ denotes taking the trace. The self-consistency equation is

$$
\Delta=\left|V_{0}\right| \cdot \operatorname{Tr} \hat{F}\left(\mathbf{r}, \mathbf{r}, \tau=\tau^{\prime}\right) .
$$

Equation system (12), (14), (15), (16) is a complete equation system for order parameter.

\section{Inhomogeneous Pairing Near Critical Transition Surface}

We solve the system of equations for the order parameter $\Delta(\mathbf{r})$ near the critical transition surface by the perturbation theory. From (14), we obtain

$$
\begin{aligned}
\hat{G}_{++} & =\hat{G}_{++}^{(0)}+\hat{G}_{++}^{(0)} \hat{\Delta} \hat{G}_{--}^{(0)} \hat{\Delta}^{+} \hat{G}_{++} ; \\
\hat{G}_{--} & =\hat{G}_{--}^{(0)}+\hat{G}_{--}^{(0)} \hat{\Delta}^{+} \hat{G}_{++}^{(0)} \hat{\Delta} \hat{G}_{--} \\
\hat{F}^{+} & =-\hat{G}_{--}^{(0)} \hat{\Delta}^{+}\left[\hat{G}_{++}^{(0)}+\hat{G}_{++}^{(0)} \hat{\Delta} \hat{G}_{--}^{(0)} \hat{\Delta}_{0}^{+} \hat{G}_{++}\right] ; \\
\hat{F} & =-\hat{G}_{++}^{(0)} \hat{\Delta}\left[\hat{G}_{--}^{(0)}+\hat{G}_{--}^{(0)} \hat{\Delta}^{+} \hat{G}_{++}^{(0)} \hat{\Delta} \hat{G}_{--}\right] .
\end{aligned}
$$

Such perturbation procedure can be easily continued up to the infinity. The behavior of the inhomogeneous order parameter near the critical surface is singular. As a result, a partial summation of "dangerous" terms should be made in the expansion (17). To do this, we express the order parameter $\Delta(\mathbf{r})$ in the inhomogeneous state in the form of a Fourier series

$\Delta(r)=\alpha_{1} \cos ((\mathbf{Q r}) / \hbar)+\alpha_{2} \cos (3(\mathbf{Q r}) / \hbar)+\alpha_{3} \cos (5(\mathbf{Q r}) / \hbar)+\ldots$

All terms inside the fifth order perturbation theory are presented in explicit form in "Appendix". In each order of perturbation theory (see (17)), the value of separate element is determined by the product of phase factors $\exp \left(i k_{j}\left(\mathbf{Q} \mathbf{r}_{j}\right) / \hbar\right)\left(k_{j}\right.$-integer number). For the term of function $\operatorname{Tr} \hat{F}\left(\mathbf{r}, \mathbf{r}, \tau=\tau^{\prime}\right)$, proportional to $\cos ((\mathbf{Q r}) / \hbar)$, the largest value in each order of perturbation theory arises only from correlated product with phase factor equal to $\exp \left\{i \mathbf{Q}\left[\left(\mathbf{r}_{1}-\mathbf{r}_{2}\right)+\cdots+\left(\mathbf{r}_{2 K-1}-\mathbf{r}_{2 K}\right)\right]+\mathbf{r}_{2 K+1}\right\}$ (or to complex conjugate). For the term of function $\operatorname{Tr} \hat{F}(\mathbf{r}, \mathbf{r}, \tau=$ $\left.\tau^{\prime}\right)$ proportional to $\cos (3(\mathbf{Q r}) / \hbar)$, the largest value in each order of perturbation theory arises from correlated product with phase factor of type $\exp \left\{i \mathbf{Q}\left[r_{1}+\left(\mathbf{r}_{2}-\mathbf{r}_{3}\right)+\cdots+\right.\right.$ $\left.\left.\left(\mathbf{r}_{2 K-2}-\mathbf{r}_{2 K-1}\right)\right]+\mathbf{r}_{2 K}+\mathbf{r}_{2 K+1}\right\}$ (or complex conjugate). For other terms, similar rules exist also.

Partial summation over these terms can be made in all orders of perturbation theory. As result to the function $F_{1}\left(\mathbf{r}, \mathbf{r}, \tau=\tau^{\prime}\right)$, we obtain the next value (see "Appendix")

$$
\begin{aligned}
F_{1}\left(\mathbf{r}, \mathbf{r}, \tau=\tau^{\prime}\right)= & T \sum_{\omega}\left|\chi_{0}(z)\right|^{2} \int_{-\infty}^{+\infty} \frac{m d \xi}{(2 \pi \hbar)^{2}} \int_{0}^{2 \pi} d \varphi \\
& \left\{\frac{\alpha_{1}}{2} \cos \left(\frac{(\mathbf{Q r})}{\hbar}\right)\left(\frac{1}{\xi^{2}-\left(i \omega-\frac{(\mathbf{P Q})}{2 m}-\lambda\right)^{2}+\alpha_{1}^{2} / 4}+\frac{1}{\xi^{2}-\left(i \omega+\frac{(\mathbf{P Q})}{2 m}-\lambda\right)^{2}+\alpha_{1}^{2} / 4}\right)\right. \\
& +\frac{\alpha_{2}}{2} \cos \left(\frac{3(\mathbf{Q r})}{\hbar}\right)\left(\frac{1}{\xi^{2}-\left(i \omega-\frac{3(\mathbf{P Q})}{2 m}-\lambda\right)^{2}}+\frac{1}{\xi^{2}-\left(i \omega+\frac{3(\mathbf{P Q})}{2 m}-\lambda\right)^{2}}\right) \\
& \left.+\frac{\alpha_{3}}{2} \cos \left(\frac{5(\mathbf{Q r})}{\hbar}\right)\left(\frac{1}{\xi^{2}-\left(i \omega-\frac{5(\mathbf{P Q})}{2 m}-\lambda\right)^{2}}+\frac{1}{\xi^{2}-\left(i \omega+\frac{5(\mathbf{P Q})}{2 m}-\lambda\right)^{2}}\right)\right\} \\
& -T \sum_{\omega} \chi_{0}(z) \chi_{0}^{*}(z) \int_{-\infty}^{+\infty} \frac{m d \xi}{(2 \pi \hbar)^{2}} \int_{0}^{2 \pi} d \varphi
\end{aligned}
$$




$$
\begin{aligned}
& \left\{\frac { \alpha _ { 1 } ^ { 3 } } { 8 } \operatorname { c o s } ( \frac { 3 ( \mathbf { Q r } ) } { \hbar } ) \left(\frac{1}{\xi^{2}-\left(i \omega-\frac{3(\mathbf{P Q})}{2 m}-\lambda\right)^{2}} \cdot \frac{1}{\xi^{2}-\left(i \omega+\frac{(\mathbf{P Q})}{2 m}-\lambda\right)^{2}+\alpha_{1}^{2} / 4}\right.\right. \\
& \left.+\frac{1}{\xi^{2}-\left(i \omega+\frac{3(\mathbf{P Q})}{2 m}-\lambda\right)^{2}} \cdot \frac{1}{\xi^{2}-\left(i \omega-\frac{(\mathbf{P Q})}{2 m}-\lambda\right)^{2}+\alpha_{1}^{2} / 4}\right) \\
& +\frac{\alpha_{1}^{3}}{4} \cos \left(\frac{(\mathbf{Q r})}{\hbar}\right)\left[\frac{1}{\left(-i \omega+\xi-\frac{3(\mathbf{P Q})}{2 m}+\lambda\right)\left(i \omega+\xi-\frac{(\mathbf{P Q})}{2 m}-\lambda\right)\left(\xi^{2}-\left(i \omega-\frac{(\mathbf{P Q})}{2 m}-\lambda\right)^{2}+\alpha_{1}^{2} / 4\right)}\right. \\
& \left.\left.+\frac{1}{\left(-i \omega+\xi+\frac{3(\mathbf{P Q})}{2 m}+\lambda\right)\left(i \omega+\xi+\frac{(\mathbf{P Q})}{2 m}-\lambda\right)\left(\xi^{2}-\left(i \omega+\frac{(\mathbf{P Q})}{2 m}-\lambda\right)^{2}+\alpha_{1}^{2} / 4\right)}\right]\right]
\end{aligned}
$$

here (PQ) is a scalar product of vector $\mathbf{P}$ on Fermi surface and vector $\mathbf{Q}, \varphi$ is angle between $\mathbf{P}$ and $\mathbf{H}, \lambda=\lambda_{+}$.

Terms $F_{2}, F_{3}$ can be considered as a small correction to the quantity $\operatorname{Tr} \hat{F}\left(r, r, \tau=\tau^{\prime}\right)$. As a result, general equation to the order parameter near critical transition surface is

$\alpha_{1} \cos \left(\frac{(\mathbf{Q r})}{\hbar}\right)+\alpha_{2} \cos \left(\frac{3(\mathbf{Q r})}{\hbar}\right)=\left|V_{0}\right| \cdot F_{1}$

Equations (19), (20) enable us to obtain expressions for free energy $F$ on the subspace of the periodic function (18). The free energy $F$ is in such a case a function of the parameters $\left\{\alpha_{1}, \alpha_{2} ; \ldots ; P Q / 2 m\right\}$. For any value of temperature $T$, the parameter $P Q / 2 m$ can be found from the equation

$\frac{\partial F}{\partial(P Q / 2 m)}=0$.

here $P=P_{F}$.

The system of equations (20) itself can be found from extremum condition of functional $F$ over parameters $\left\{\alpha_{1}, \alpha_{2} ; \ldots\right\}$, i.e.,

$\frac{\partial F}{\partial \alpha_{j}}=0, \quad\left\{\alpha_{j}=1,2, \ldots\right\}$.

Below, we will consider low-temperature region $T \ll$ $P Q / 2 m$.

Note that integrals in (19) can be written down in a form more simple for integration. To do this, we note that in a low-temperature region the main contribution to the integrals arises from regions of $\varphi$ close to $\pi / 2$, with $a_{-}$in denominator of integrals, or in the regions near the points $\left\{\varphi_{0}=-\arcsin \left(\left|R_{2}\right|\right) ; \varphi_{1}=-\pi-\varphi_{0}\right\}$ if in denominator of integrals stay $a_{+}$. The quantities $a_{ \pm}$are equal to

$a_{ \pm}=\frac{p_{F} Q}{2 m} \sin \varphi \pm \lambda$.

In the vicinity of point $\varphi=\frac{\pi}{2}$, we have

$$
\begin{aligned}
\varphi & =\frac{\pi}{2}+\delta \varphi ; \sin \varphi=1-\frac{\delta \varphi^{2}}{2} ; \\
a_{-} & =-\frac{h}{2 \sqrt{\gamma_{1}}}\left\{\frac{1-R_{2}}{2}(\delta \varphi)^{2}+\frac{\delta \gamma_{1}}{\gamma_{1}}\right\} ; \frac{h}{2 \sqrt{\gamma_{1}}} \frac{\delta \gamma_{1}}{\gamma_{1}}=-\pi T \beta
\end{aligned}
$$

The quantities $\left\{\delta \gamma_{1} / \gamma_{1}, \beta\right\}$ have been found in paper [4]. For $T=0$, eight branches are found in paper [3] with different values of $\mathbf{Q}$ on the plane of parameters $\left(\gamma_{1}, \gamma_{2}\right)$, where

$$
\gamma_{1}=\left(\frac{2 h}{v_{F} Q}\right)^{2}, \gamma_{2}=\left(\frac{\tilde{\alpha} \epsilon_{F}}{h}\right)^{2}
$$

Optimal are branches I, II. These branches are parametrized by one parameter $R_{2}\left(-1<R_{2}<-1 / 2\right)$. Parameter $\gamma_{2}$ we consider as given. And for small values of $T$ (not equal to 0 ), we can put [4]

$$
\frac{\delta \gamma_{1}}{\gamma_{1}}=-\beta Z \frac{1-R_{2}}{2}
$$


The quantity $\beta$ is dimensionless function of $Z$. Definition of function $Z$ and exact equation for $\beta$ are given in (49). In main approximation, we have

$\beta=\frac{1}{\pi} \ln \left(\frac{\pi}{\left(1-R_{2}\right) \sqrt{Z}}\right)$

In the vicinity of point $\varphi_{0}=-\arcsin \left(\left|R_{2}\right|\right)$, we have

$$
\begin{aligned}
\sin \varphi & =R_{2}+\sqrt{1-R_{2}^{2}} \delta \varphi ; \\
a_{+} & =\frac{h}{\sqrt{\gamma_{1}}}\left\{-\sqrt{1-R_{2}^{2}} \frac{1-R_{2}}{2 R_{2}} \delta \varphi-\frac{\delta \gamma_{1} R_{2}}{2 \gamma_{1}}\right\} .
\end{aligned}
$$

And in the vicinity of point $\varphi_{1}=-\pi+\arcsin \left(\left|R_{2}\right|\right)$, we obtain

$$
\begin{aligned}
\sin \varphi & =R_{2}-\sqrt{1-R_{2}^{2}} \delta \varphi ; \\
a_{+} & =\frac{h}{\sqrt{\gamma_{1}}}\left\{\sqrt{1-R_{2}^{2}} \frac{1-R_{2}}{2 R_{2}} \delta \varphi-\frac{\delta \gamma_{1} R_{2}}{2 \gamma_{1}}\right\} .
\end{aligned}
$$

Now, we define three integrals $\left\{J, J_{1}, J_{2}\right\}$ that are parts of integrals in (19).

$$
\begin{aligned}
J= & T \sum_{\omega}\left(\chi_{0}(z)\right)^{2} \int_{-\infty}^{\infty} \frac{d \xi}{(2 \pi \hbar)^{2}} \int_{0}^{2 \pi} d \varphi\left\{\left(\frac{1}{\xi^{2}+\frac{\alpha_{1}^{2}}{4}-\left(i \omega-a_{+}\right)^{2}}+\frac{1}{\xi^{2}+\frac{\alpha_{1}^{2}}{4}-\left(i \omega+a_{-}\right)^{2}}\right)\right. \\
& \left.-\left(\frac{1}{\xi^{2}-\left(i \omega-a_{+}\right)^{2}}+\frac{1}{\xi^{2}-\left(i \omega+a_{-}\right)^{2}}\right)\right\} \\
J_{1}= & T \sum_{\omega}\left(\chi_{0}(z)\right)^{2} \int_{-\infty}^{\infty} \frac{d \xi}{(2 \pi \hbar)^{2}} \int_{0}^{2 \pi} d \varphi\left\{\frac{\xi^{2}-\left(i \omega+a_{-}\right)^{2}}{\xi^{2}-\left(i \omega-\frac{3(\mathbf{P Q})}{2 m}-\lambda\right)^{2}}\left(\frac{1}{\xi^{2}-\left(i \omega+a_{-}\right)^{2}}-\frac{1}{\xi^{2}+\frac{\alpha_{1}^{2}}{4}-\left(i \omega+a_{-}\right)^{2}}\right)\right. \\
& \left.+\frac{\xi^{2}-\left(i \omega-a_{+}\right)^{2}}{\xi^{2}-\left(i \omega+\frac{3(\mathbf{P Q})}{2 m}-\lambda\right)^{2}} \int_{-\left(i \omega-a_{+}\right)^{2}} \frac{\xi^{2}+\frac{\alpha_{1}^{2}}{4}-\left(i \omega-a_{+}\right)^{2}}{\xi^{2}-(i \omega)}\right) \\
J_{2}= & T \sum_{\omega}\left(\chi_{0}(z)\right)^{2} \int_{-\infty}^{\infty} \frac{d \xi}{(2 \pi \hbar)^{2}} \int_{0}^{2 \pi} d \varphi\left\{\frac{-i \omega+\xi+a_{+}}{\left(-i \omega+\xi-\frac{3(\mathbf{P Q})}{2 m}(\lambda)\right.}\left(\frac{1}{\xi^{2}-\left(i \omega-a_{+}\right)^{2}}-\frac{1}{\xi^{2}+\frac{\alpha_{1}^{2}}{4}-\left(i \omega-a_{+}\right)^{2}}\right)\right. \\
& \left.+\frac{-i \omega+\xi-a_{-}}{\left(-i \omega+\xi+\frac{3(\mathbf{P Q})}{2 m}+\lambda\right)^{2}}\left(\frac{1}{\xi^{2}-\left(i \omega+a_{-}\right)^{2}}-\frac{1}{\xi^{2}+\frac{\alpha_{1}^{2}}{4}-\left(i \omega+a_{-}\right)^{2}}\right)\right\}
\end{aligned}
$$

More simple for calculations are derivatives of integrals $\left\{J, J_{1}, J_{2}\right\}$ over parameter $\alpha_{1}$. With help from (24), (28), (29), (30), we obtain

$$
\begin{aligned}
\frac{\partial J}{\partial \alpha_{1}}= & T \sum_{\omega}\left|\chi_{0}\right|^{2} \int_{-\infty}^{\infty} \frac{d \xi}{(2 \pi \hbar)^{2}} \frac{\partial}{\partial \alpha_{1}}\left\{\left(\frac{2 \sqrt{\gamma_{1}}}{h} \frac{2}{1-R_{2}}\right)^{1 / 2} \int_{-\infty}^{\infty} d t \frac{1}{\xi^{2}+\frac{\alpha_{1}^{2}}{4}-\left[i \omega-\left(t^{2}+\frac{h}{2 \sqrt{\gamma_{1}}} \frac{\delta \gamma_{1}}{\gamma_{1}}\right)\right]^{2}}\right. \\
& \left.-2\left(\frac{2\left|R_{2}\right|}{1-R_{2}} \frac{\sqrt{\gamma_{1}}}{h \sqrt{1-R_{2}^{2}}}\right) \int_{-\infty}^{\infty} d t \frac{1}{\left(t-i \omega+\sqrt{\xi^{2}+\alpha_{1}^{2} / 4}\right)\left(t-i \omega-\sqrt{\xi^{2}+\alpha_{1}^{2} / 4}\right)}\right\}
\end{aligned}
$$


The last integral in (31) is equal to 0 . That is, the main contribution arises only from term with $a_{-}$in denominator.
The same situation takes place for integrals $\left\{J_{1}, J_{2}\right\}$. We have

$$
\begin{aligned}
-\frac{2}{\alpha_{1}} \frac{\partial}{\partial \alpha_{1}}\left(\frac{2}{\alpha_{1}} \frac{\partial J_{1}}{\partial \alpha_{1}}\right)= & T \sum_{\omega}\left|\chi_{0}(z)\right|^{2} \int_{-\infty}^{\infty} \frac{d \xi}{(2 \pi \hbar)^{2}} \int_{0}^{2 \pi} d \varphi \frac{\xi^{2}-\left(i \omega+a_{-}\right)^{2}}{\xi^{2}-\left(i \omega-\frac{3(\mathbf{P Q})}{2 m}-\lambda\right)^{2}} \\
& \cdot \frac{2}{\left(\xi^{2}+\frac{\alpha_{1}^{2}}{4}-\left(i \omega+a_{-}\right)^{2}\right)^{3}} \\
-\frac{2}{\alpha_{1}} \frac{\partial}{\partial \alpha_{1}}\left(\frac{2}{\alpha_{1}} \frac{\partial J_{2}}{\partial \alpha_{1}}\right)= & T \sum_{\omega}\left|\chi_{0}(z)\right|^{2} \int_{-\infty}^{\infty} \frac{d \xi}{(2 \pi \hbar)^{2}} \int_{0}^{2 \pi} d \varphi \frac{-i \omega+\xi-a_{-}}{-i \omega+\xi+\frac{3(\mathbf{P Q})}{2 m}+\lambda} \\
& \cdot \frac{2}{\left(\xi^{2}+\frac{\alpha_{1}^{2}}{4}-\left(i \omega+a_{-}\right)^{2}\right)^{3}} \cdot
\end{aligned}
$$

Main contribution in the integral value arises from the point $\left(\varphi=\frac{\pi}{2}\right)$, where we will put

$\varphi=\frac{\pi}{2}+\delta \varphi, \quad \sin \varphi=1-(\delta \varphi)^{2} / 2, \quad \delta \varphi=\left(\frac{2 \sqrt{\gamma_{1}}}{h} \frac{2}{1-R_{2}}\right)^{1 / 2} t$.

Equations (31), (32), and (33) enable us to calculate the value of all integrals $\left(J, J_{1}, J_{2}\right)$ and complete estimation of equation for order parameter in low-temperature region.

\section{Subregion of Low-Temperature Region $\alpha_{1}>2 \pi T \beta$}

The low-temperature region $P_{F} Q / m \gg \pi T$ decays on two subregions: $\alpha_{1}>2 \pi T \beta$ and $\alpha_{1} \ll 2 \pi T \beta$. We will consider first the region $\alpha_{1}>2 \pi T \beta$. In this region, we obtain from (31)

$$
\begin{aligned}
\frac{\partial J}{\partial \alpha_{1}}= & \left\langle\left|\chi_{0}(z)\right|^{2}\right\rangle\left(\frac{2 \sqrt{\gamma_{1}}}{h} \frac{2}{1-R_{2}}\right)^{1 / 2} \frac{\partial}{\partial \alpha_{1}} \int_{-\infty}^{\infty} \frac{d \xi}{(2 \pi \hbar)^{2}} \int_{-\infty}^{\infty} d t \\
& \times\left\{\frac{1}{2 \pi} \int_{-\infty}^{\infty} d \omega \frac{1}{\xi^{2}+\frac{\alpha_{1}^{2}}{4}+\left(\omega+i\left(t^{2}-\pi T \beta\right)\right)^{2}}\right. \\
& +\left[\frac{T}{2 i} \underset{\underset{.}{\longleftarrow} d}{\longleftarrow} d y(\operatorname{tg}(\pi y)-i)+\frac{T}{2 i} \int d y(\operatorname{tg}(\pi y)+i)\right] \\
& \left.\times \frac{1}{\xi^{2}+\frac{\alpha_{1}^{2}}{4}-\left[i 2 \pi T y-\left(t^{2}-\pi T \beta\right)\right]^{2}}\right\}
\end{aligned}
$$

Here the arrow means the integration contour and points indicate the poles of $\operatorname{tg}(\pi y)$.
In the range $\alpha_{1}>2 \pi T \beta$, we obtain from (34)

$$
\begin{aligned}
\frac{\partial J}{\partial \alpha_{1}}= & \left\langle\left|\chi_{0}(z)\right|^{2}\right\rangle\left(\frac{2 \sqrt{\gamma_{1}}}{h} \frac{2}{1-R_{2}}\right)^{1 / 2} \int_{-\infty}^{\infty} \frac{d \xi}{(2 \pi \hbar)^{2}} \frac{\partial}{\partial \alpha_{1}} \\
& \left\{\frac{R}{\sqrt{\xi^{2}+\alpha_{1}^{2} / 4}}-\int_{0}^{R} \frac{d t}{\sqrt{\xi^{2}+\alpha_{1}^{2} / 4}} \frac{\exp \left(-\left(R^{2}-t^{2}\right) / T\right)}{1+\exp \left(-\left(R^{2}-t^{2}\right) / T\right)}\right. \\
& -\int_{0}^{\infty} \frac{d t}{\sqrt{\xi^{2}+\alpha_{1}^{2} / 4}} \frac{\exp \left(-\left(t^{2}+\sqrt{\xi^{2}+\frac{\alpha_{1}^{2}}{4}}-\pi T \beta\right) / T\right)}{1+\exp \left(-\left(t^{2}+\sqrt{\xi^{2}+\frac{\alpha_{1}^{2}}{4}}-\pi T \beta\right) / T\right)} \\
& \left.+\int_{R}^{\infty} \frac{d t}{\sqrt{\xi^{2}+\alpha_{1}^{2} / 4}} \frac{\exp \left(-\left(t^{2}-R^{2}\right) / T\right)}{1+\exp \left(-\left(t^{2}-R^{2}\right) / T\right)}\right\}
\end{aligned}
$$

where $R=\left(\sqrt{\xi^{2}+\alpha_{1}^{2} / 4}+\pi T \beta\right)^{1 / 2}$.

The second and fourth terms in (35) strongly cancel each other. Expansion is going over powers of $\left(T / R^{2}\right)$. As result, we obtain

$$
\begin{aligned}
& -\int_{0}^{R} d t \frac{\exp \left(-\left(R^{2}-t^{2}\right) / T\right)}{1+\exp \left(-\left(R^{2}-t^{2}\right) / T\right)} \\
& +\int_{R}^{\infty} d t \frac{\exp \left(-\left(t^{2}-R^{2}\right) / T\right)}{1+\exp \left(-\left(t^{2}-R^{2}\right) / T\right)}=-\frac{T^{2}}{4 \cdot R^{3}} \zeta_{(2)}, \\
& \int_{-\infty}^{\infty} d \xi \int_{0}^{\infty} \frac{d t}{\sqrt{\xi^{2}+\alpha_{1}^{2} / 4}} \frac{\exp \left(-\left(t^{2}+\sqrt{\xi^{2}+\alpha_{1}^{2} / 4}-\pi T \beta\right) / T\right)}{1+\exp \left(-\left(t^{2}+\sqrt{\xi^{2}+\alpha_{1}^{2} / 4}-\pi T \beta\right) / T\right)} \\
& =\frac{\pi T}{\sqrt{\alpha_{1}}} \ln \left(1+\exp \left(-\left(\frac{\alpha_{1}}{2 T}-\pi \beta\right)\right)\right)
\end{aligned}
$$


Final answer for the function $J$ in the region $\alpha_{1}>2 \pi T \beta$ is

$$
\begin{aligned}
J= & \left\langle\left|\chi_{0}(z)\right|^{2}\right\rangle\left(\frac{2 \sqrt{\gamma_{1}}}{h} \frac{2}{1-R_{2}}\right)^{1 / 2} \frac{1}{(2 \pi \hbar)^{2}} \\
& \cdot\left\{J^{(2)}-\frac{\pi T}{\sqrt{\alpha_{1}}} \ln \left[1+\exp \left(-\left(\frac{\alpha_{1}}{2 T}-\pi \beta\right)\right)\right]\right. \\
& \left.-\frac{\zeta_{(2)} T^{2}}{2} \int_{0}^{\infty} \frac{d \xi}{R^{3} \sqrt{\xi^{2}+\frac{\alpha_{1}^{2}}{4}}}\right\}
\end{aligned}
$$

here $\zeta_{(x)}$-is Riemann $\zeta$ function, and function $J^{(2)}$ is given by equation

$$
\begin{aligned}
\frac{\partial J^{(2)}}{\partial \alpha_{1}} & =2 \int_{0}^{\infty} d \xi \frac{\partial}{\partial \alpha_{1}}\left(\frac{R}{\sqrt{\xi^{2}+\alpha_{1}^{2} / 4}}\right) \\
& =-\frac{1}{\sqrt{2 \alpha_{1}}} \int_{0}^{\infty} d y \frac{\sqrt{y^{2}+1}+4 \pi T \beta / \alpha_{1}}{\left(y^{2}+1\right)^{3 / 2}\left(\sqrt{y^{2}+1}+2 \pi T \beta / \alpha_{1}\right)^{1 / 2}} .
\end{aligned}
$$

It has series expansion over parameter $\left(2 \pi T \beta / \alpha_{1}\right)$

$J^{(2)}=-\sqrt{2 \alpha_{1}} C_{1}+\sqrt{2 \alpha_{1}} \cdot \frac{3 \pi T \beta}{\alpha_{1}} C_{2}+\ldots$

with coefficients $C_{1}, C_{2}$ equal to

$$
\begin{aligned}
& C_{1}=\int_{0}^{\infty} \frac{d y}{\left(y^{2}+1\right)^{5 / 4}}=\sqrt{2}\left[(2-\sqrt{2})+\sum_{K=1}^{\infty} B_{K}\right]=1.198134 ; \\
& B_{1}=\frac{1}{3}, B_{K}=-B_{K-1} \frac{(K-5 / 4)(K-1 / 2)}{K(K-1 / 4)} ; \\
& C_{2}=\int_{0}^{\infty} \frac{d y}{\left(y^{2}+1\right)^{7 / 4}}=\frac{\sqrt{2}}{3} \sum_{K=0}^{\infty} \tilde{B}_{K}=0.874015
\end{aligned}
$$

$$
\begin{aligned}
\tilde{B}_{0}= & 2, \tilde{B}_{K}=-\tilde{B}_{K-1} \cdot \frac{(K-1 / 2)}{K} \cdot \frac{(K-3 / 4)}{K+1 / 4} ; K \geqslant 1 \\
& \int_{0}^{\infty} \frac{d y}{\left(y^{2}+1\right)^{9 / 4}}=\frac{3}{5} C_{1} ; \int_{0}^{\infty} \frac{d y}{\left(y^{2}+1\right)^{11 / 4}}=\frac{5}{7} C_{2} ; \ldots
\end{aligned}
$$

The last integral in (37) also has series expansion over parameter $2 \pi T \beta / \alpha_{1}$.

$$
\begin{aligned}
\int_{0}^{\infty} \frac{d \xi}{R^{3} \sqrt{\xi^{2}+\alpha_{1}^{2} / 4}} & =\left(\frac{2}{\alpha_{1}}\right)^{3 / 2} \int_{0}^{\infty} \frac{d y}{\left(y^{2}+1\right)^{5 / 4}\left(1+\frac{2 \pi T \beta}{\alpha_{1} \sqrt{y^{2}+1}}\right)^{3 / 2}} \\
& =\left(\frac{2}{\alpha_{1}}\right)^{3 / 2}\left[C_{1}-\frac{3 \pi T \beta}{\alpha_{1}} C_{2} \ldots\right]
\end{aligned}
$$

In the same way, we calculate the value of integrals $\left\{J_{1}, J_{2}\right\}$ from (32). We have

$$
\begin{aligned}
J_{1}= & \left\langle\left|\chi_{0}(z)\right|^{2}\right\rangle \cdot\left(\frac{2 \sqrt{\gamma_{1}}}{h} \frac{2}{1-R_{2}}\right)^{1 / 2} \frac{1}{(2 \pi \hbar)^{2}}\left(\frac{m}{2 P_{F} Q}\right)^{2} \\
& \left\{\frac { \alpha _ { 1 } ^ { 2 } } { 4 } \left(\frac{T^{2} \zeta_{(2)}}{2} \int_{0}^{\infty} \frac{d \xi}{R^{3} \sqrt{\xi^{2}+\alpha_{1}^{2} / 4}}\right.\right. \\
& \left.\left.+\frac{\pi T}{\sqrt{\alpha_{1}}} \ln \left[1+\exp \left(-\left(\frac{\alpha_{1}}{2 T}-\pi \beta\right)\right)\right]\right)+J_{1}^{(2)}\right\}
\end{aligned}
$$

where function $J_{1}^{(2)}$ is solution of equation

$$
\begin{aligned}
\frac{2}{\alpha_{1}} \frac{\partial}{\partial \alpha_{1}}\left(\frac{2}{\alpha_{1}} \frac{\partial J_{1}^{(2)}}{\partial \alpha_{1}}\right)= & 2\left(\frac{2}{\alpha_{1}}\right)^{3 / 2} \int_{0}^{\infty} \frac{d y}{\left(y^{2}+1\right)^{5 / 2}} \\
& \times\left(y^{2}+1 / 4\right)\left(\sqrt{y^{2}+1}+\frac{2 \pi T \beta}{\alpha_{1}}\right)^{1 / 2}
\end{aligned}
$$


Function $J_{1}^{(2)}$ has series expansion over parameter $2 \pi T \beta / \alpha_{1}$. We obtain from (43)

$J_{1}^{(2)}=\frac{22}{50} C_{1} \sqrt{2} \alpha_{1}^{5 / 2}-\frac{13}{21} \sqrt{2} \pi T \beta C_{2} \cdot \alpha_{1}^{3 / 2}+\ldots$

Function $J_{2}$ is equal to (in the range $\alpha_{1}>2 \pi T \beta$ )

$$
\begin{aligned}
J_{2}= & -\left\langle\left|\chi_{0}(z)\right|^{2}\right\rangle \frac{1}{(2 \pi \hbar)^{2}}\left(\frac{2 \sqrt{\gamma_{1}}}{h} \frac{2}{1-R_{2}}\right)^{1 / 2} \frac{m}{2 P_{F} Q} \\
& \left\{-\frac{T^{2} \zeta_{(2)}}{2} \int_{0}^{\infty} \frac{d \xi}{R^{3}}+\frac{\pi T \sqrt{\alpha_{1}}}{2} \ln \right. \\
& {\left.\left[1+\exp \left(-\left(\frac{\alpha_{1}}{2 T}-\pi \beta\right)\right)\right]+J_{2}^{(2)}\right\} ; J_{2}^{(2)}=0 }
\end{aligned}
$$

The integral in (45) is equal to

$$
\begin{aligned}
\int_{0}^{\infty} \frac{d \xi}{R^{3}} & =\left(\frac{2}{\alpha_{1}}\right)^{1 / 2} \int_{0}^{\infty} \frac{d y}{\left(\sqrt{y^{2}+1}+\frac{2 \pi T \beta}{\alpha_{1}}\right)^{3 / 2}} \\
\int_{0}^{\infty} \frac{d y}{\left(y^{2}+1\right)^{3 / 4}} & =3 \int_{0}^{\infty} \frac{d y}{\left(y^{2}+1\right)^{7 / 4}}=3 C_{2}
\end{aligned}
$$

\section{Subregion $\alpha_{1}<2 \pi T \beta$}

Consider now the low-temperature subregion restricted by condition $\alpha_{1}<2 \pi T \beta$. In this case, we will use the equation, estimated in [4].

$$
\begin{aligned}
& \frac{i}{2} \int_{-\infty}^{\infty} d t\left\{\psi^{\prime}\left(\frac{1}{2}+\frac{i}{2}\left(t^{2}-\beta\right)\right)-\psi^{\prime}\left(\frac{1}{2}-\frac{i}{2}\left(t^{2}-\beta\right)\right)\right\} \\
& =2 \pi^{2} \sum_{K=1}^{\infty}(-)^{K+1} \sqrt{K} \exp (-\pi K \beta) \\
& \int_{-\infty}^{\infty} d t\left\{\psi^{\prime \prime}\left(\frac{1}{2}+\frac{i}{2}\left(t^{2}-\beta\right)\right)+\psi^{\prime \prime}\left(\frac{1}{2}-\frac{i}{2}\left(t^{2}-\beta\right)\right)\right\} \\
& =8 \pi^{3} \sum_{K=1}^{\infty}(K)^{3 / 2}(-)^{K} \exp (-\pi K \beta)
\end{aligned}
$$

In main approximation over parameter $\alpha_{1} /(2 \pi T \beta)$, we obtain from (30)

$$
\begin{aligned}
J= & \frac{\alpha_{1}^{2}}{32} \frac{\sqrt{\pi T}}{(2 \pi T)^{2}}\left\langle\left|\chi_{0}(z)\right|^{2}\right\rangle \frac{1}{(2 \pi \hbar)^{2}}\left(\frac{2 \sqrt{\gamma_{1}}}{h} \frac{2}{1-R_{2}}\right)^{1 / 2} \\
& \int_{-\infty}^{\infty} d t\left\{\psi^{\prime \prime}\left(\frac{1}{2}+\frac{i}{2}\left(t^{2}-\beta\right)\right)+\psi^{\prime \prime}\left(\frac{1}{2}-\frac{i}{2}\left(t^{2}-\beta\right)\right)\right\} .
\end{aligned}
$$

The quantity $\beta$ is solution of equation [4]

$$
\sum_{K=1}^{\infty}(-)^{K+1} \sqrt{K} \exp (-\pi K \beta)=\frac{1-R_{2}}{\pi} \sqrt{Z} \quad ; \quad Z=\frac{4 \pi T \sqrt{\gamma_{1}}}{h\left(1-R_{2}\right)} .
$$

From (47), (48), and (49), we obtain

$$
J=-\frac{\pi \alpha_{1}^{2}}{4 T}\left(\frac{\sqrt{\gamma_{1}}}{h}\right) \frac{\left\langle\left|\chi_{0}(z)\right|^{2}\right\rangle}{(2 \pi \hbar)^{2}} \quad ; \quad \alpha_{1} \ll 2 \pi T \beta .
$$

From (32), it follows that

$$
\begin{aligned}
& -\frac{2}{\alpha_{1}} \frac{\partial}{\partial \alpha_{1}}\left(\frac{2}{\alpha_{1}} \frac{\partial J_{1}}{\partial \alpha_{1}}\right)=J \cdot \frac{8}{\alpha_{1}^{2}}\left(\frac{m}{2 P_{F} Q}\right)^{2} ; \\
& J_{1}=\frac{\pi \alpha_{1}^{4}}{16 T}\left(\frac{m}{2 P_{F} Q}\right)^{2} \frac{\left\langle\left|\chi_{0}(z)\right|^{2}\right\rangle}{(2 \pi \hbar)^{2}}\left(\frac{\sqrt{\gamma_{1}}}{h}\right) .
\end{aligned}
$$

In the same approximation, we obtain the next equation for function $J_{2}$

$$
\begin{aligned}
J_{2}= & -\left\langle\left|\chi_{0}(z)\right|^{2}\right\rangle\left(\frac{m}{2 P_{F} Q}\right) \frac{\alpha_{1}^{2}}{16 \sqrt{\pi T}}\left(\frac{2 \sqrt{\gamma_{1}}}{h} \cdot \frac{2}{1-R_{2}}\right)^{1 / 2} \frac{1}{(2 \pi \hbar)^{2}} \frac{i}{2} \\
& \cdot \int_{-\infty}^{\infty} d t\left(\psi^{\prime}\left(\frac{1}{2}+\frac{i}{2}\left(t^{2}-\beta\right)\right)-\psi^{\prime}\left(\frac{1}{2}-\frac{i}{2}\left(t^{2}-\beta\right)\right)\right) .(52)
\end{aligned}
$$

Final expression for value of quantity $J_{2}$, following from (47) and (52), is

$$
J_{2}=-\left\langle\left|\chi_{0}\right|^{2}\right\rangle \frac{1}{(2 \pi \hbar)^{2}}\left(\frac{m}{2 P_{F} Q}\right) \frac{\pi \alpha_{1}^{2}}{2}\left(\frac{\sqrt{\gamma_{1}}}{h}\right) .
$$


As a result, in low-temperature region $\pi T \ll P_{F} Q / 2 m$, the

(20) for order parameter acquires the form

$$
\begin{aligned}
\alpha_{1} \cos \left(\frac{(\mathbf{Q r})}{\hbar}\right)+\alpha_{2} \cos \left(\frac{3(\mathbf{Q r})}{\hbar}\right)= & \left|V_{0}\right| m\left\{\cos \left(\frac{(\mathbf{Q r})}{\hbar}\right) \cdot \alpha_{1}\left(0.5 \cdot J-J_{2}\right)-\frac{\alpha_{1}}{2} J_{1} \cos \left(\frac{(3 \mathbf{Q r})}{\hbar}\right)\right\} \\
& +\frac{\left|V_{0}\right| m\left\langle\left|\chi_{0}(z)\right|^{2}\right\rangle}{4(2 \pi \hbar)^{2}}\left\{\alpha _ { 1 } \operatorname { c o s } ( \frac { ( \mathbf { Q r } ) } { \hbar } ) \left[4 \pi \ln \left(\frac{\gamma_{1}(T=0) \omega_{D}^{2}}{h^{2}}\right)\right.\right. \\
& \left.+8 \pi \ln 2-2\left(1-R_{2}\right) Z \cdot\left(1+\ln \left(\frac{\pi}{\left(1-R_{2}\right) \sqrt{Z}}\right)\right)\right] \\
& \left.+\alpha_{2} \cos \left(\frac{3(\mathbf{Q r})}{\hbar}\right)\left[4 \pi \ln \left(\frac{\gamma_{1} \omega_{D}^{2}}{9 h^{2}}\right)+8 \pi \ln 2\right]\right\}
\end{aligned}
$$

To obtain (54), we used the value of linear on $\alpha_{1}$ terms from paper [4] and next equation

$$
\begin{aligned}
& T \sum_{\omega} \int_{-\infty}^{\infty} \frac{d \xi}{(2 \pi \hbar)^{2}} \int_{0}^{2 \pi} d \varphi\left[\frac{1}{\xi^{2}-\left(i \omega-\left(\frac{3(\mathbf{P Q})}{2 m}+\lambda\right)\right)^{2}}+\frac{1}{\xi^{2}-\left(i \omega+\left(\frac{3(\mathbf{P Q})}{2 m}-\lambda\right)\right)^{2}}\right] \\
& =\frac{1}{2(2 \pi \hbar)^{2}}\left\{4 \pi \ln \left(\frac{\gamma_{1} \omega_{D}^{2}}{9 h^{2}}\right)+8 \pi \ln 2\right\} .
\end{aligned}
$$

Equation (54) gives temperature, magnetic field, and spinorbit interaction dependence of the order parameter $\Delta(\mathbf{r})$ in low-temperature region $\pi T \ll P_{F} Q / 2 m$ near the critical surface.

\section{Conclusions}

We obtain explicit expression for the order parameter in two-dimensional space (thin films) in inhomogeneous state near the critical surface in low-temperature region $\pi T \ll$ $\left(P_{F} Q\right) / 2 m$. Low-temperature region $\pi T \ll\left(P_{F} Q\right) / 2 m$ separated for two subregions: $\alpha_{1}>2 \pi T \beta$ and $\alpha_{1}<2 \pi T \beta$. In full subregion $\alpha_{1}>2 \pi T \beta$, the relative simple general equations for all coefficients are found. In this subregion, series expansion is going over parameter $2 \pi \beta T / \alpha_{1}$. All functions are singular in this subregion. Usual expansion over order parameter $\Delta$ near the critical point is going over integer powers of $\Delta$ similar to that as it takes place in Ginzburg-Landau approximation: after linear term, cubic term is going. In the considered region, next term has power $3 / 2$ (see $(37,39,50)$ ). Such singularity will present special interest for experimental investigation. Equation (34) is valid also in subregion $\alpha_{1}<2 \pi \beta T$. In this subregion, some additional restrictions arise for integration over $\xi, t$. Partially, in first integral (34), we obtain restriction from down $\pi T \beta-\sqrt{\left(\alpha_{1}\right)^{2} / 4+(\xi)^{2}}<(t)^{2}<(R)^{2}$. In subregion $\alpha_{1}<<2 \pi T \beta$, expansion of all coefficients is going over parameter $\alpha_{1} / 2 \pi T \beta$. Nontrivial key equation that enables us to relatively easily find expansion coefficients in this region is given by (47).

We present also the general equation for inhomogeneous state in full temperature region near the critical surface. The reconstruction of free energy functional on the subspace of periodic functions can be made with the help of (20).

Acknowledgements The considered problem was formulated by Prof. P. Fulde. I thank him for the permanent attention, numerous discussions, and real collaboration.

Funding Information Open access funding provided by Max Planck Society.

Open Access This article is distributed under the terms of the Creative Commons Attribution 4.0 International License (http://creativecommons. org/licenses/by/4.0/), which permits unrestricted use, distribution, and reproduction in any medium, provided you give appropriate credit to the original author(s) and the source, provide a link to the Creative Commons license, and indicate if changes were made.

\section{Appendix}

With help from (14), (17), (18), we obtain the next expression for function $\operatorname{Tr} \hat{F}\left(\mathbf{r}, \mathbf{r}, \tau=\tau^{\prime}\right)$ inside fifth order perturbation theory over quantity $\alpha_{1}$

$$
\begin{aligned}
\operatorname{Tr} \hat{F}\left(\mathbf{r}, \mathbf{r}, \tau=\tau^{\prime}\right)= & F_{1}\left(\mathbf{r}, \mathbf{r}, \tau=\tau^{\prime}\right)+F_{2}\left(\mathbf{r}, \mathbf{r}, \tau=\tau^{\prime}\right) \\
& +F_{3}\left(\mathbf{r}, \mathbf{r}, \tau=\tau^{\prime}\right)
\end{aligned}
$$


where

$$
\begin{aligned}
& F_{1}\left(\mathbf{r}, \mathbf{r}, \tau=\tau^{\prime}\right)=T \sum_{\omega} \chi_{0}(z) \chi_{0}^{*}(z) \int_{-\infty}^{\infty} \frac{m d \xi}{(2 \pi \hbar)^{2}} \int_{0}^{2 \pi} d \varphi \\
& \left\{\frac { \alpha _ { 1 } } { 2 } \operatorname { c o s } ( \frac { ( \mathbf { Q r } ) } { \hbar } ) \left[\frac { 1 } { \xi ^ { 2 } - ( i \omega - \frac { ( \mathbf { P Q } ) } { 2 m } - \lambda ) ^ { 2 } } \left(1-\frac{\alpha_{1}^{2} / 4}{\xi^{2}-\left(i \omega-\frac{(\mathbf{P Q})}{2 m}-\lambda\right)^{2}}\right.\right.\right. \\
& \left.+\frac{\left(\alpha_{1}^{2} / 4\right)^{2}}{\left(\xi^{2}-\left(i \omega-\frac{(\mathbf{P Q})}{2 m}-\lambda\right)^{2}\right)^{2}}\right)+\frac{1}{\xi^{2}-\left(i \omega+\frac{(\mathbf{P Q})}{2 m}-\lambda\right)^{2}} \\
& \left.\cdot\left(1-\frac{\alpha_{1}^{2} / 4}{\xi^{2}-\left(i \omega+\frac{(\mathbf{P Q})}{2 m}-\lambda\right)^{2}}+\frac{\left(\alpha_{1}^{2} / 4\right)^{2}}{\left(\xi^{2}-\left(i \omega+\frac{(\mathbf{P Q})}{2 m}-\lambda\right)^{2}\right)^{2}}\right)\right] \\
& +\frac{\alpha_{2}}{2} \cos \left(\frac{3(\mathbf{Q r})}{\hbar}\right)\left(\frac{1}{\xi^{2}-\left(i \omega-\frac{3(\mathbf{P Q})}{2 m}-\lambda\right)^{2}}+\frac{1}{\xi^{2}-\left(i \omega+\frac{3(\mathbf{P Q})}{2 m}-\lambda\right)^{2}}\right) \\
& \left.+\frac{\alpha_{3}}{2} \cos \left(\frac{5(\mathbf{Q r})}{\hbar}\right)\left(\frac{1}{\xi^{2}-\left(i \omega-\frac{5(\mathbf{P Q})}{2 m}-\lambda\right)^{2}}+\frac{1}{\xi^{2}-\left(i \omega+\frac{5(\mathbf{P Q})}{2 m}-\lambda\right)^{2}}\right)\right\} \\
& -T \sum_{\omega} \chi_{0}(z) \chi_{0}^{*}(z) \int_{-\infty}^{\infty} \frac{m d \xi}{(2 \pi \hbar)^{2}} \int_{0}^{2 \pi} d \varphi \\
& \left\{\frac { \alpha _ { 1 } ^ { 3 } } { 8 } \operatorname { c o s } ( \frac { 3 ( \mathbf { Q r } ) } { \hbar } ) \left[\frac{1}{\xi^{2}-\left(i \omega-\frac{3(\mathbf{P Q})}{2 m}-\lambda\right)^{2}}\left(\frac{1}{\xi^{2}-\left(i \omega+\frac{(\mathbf{P Q})}{2 m}-\lambda\right)^{2}}-\frac{\alpha_{1}^{2} / 4}{\left(\xi^{2}-\left(i \omega+\frac{(\mathbf{P Q})}{2 m}-\lambda\right)^{2}\right)^{2}}\right)\right.\right. \\
& \left.+\frac{1}{\xi^{2}-\left(i \omega+\frac{3(\mathbf{P Q})}{2 m}-\lambda\right)^{2}} \cdot\left(\frac{1}{\xi^{2}-\left(i \omega-\frac{(\mathbf{P Q})}{2 m}-\lambda\right)^{2}}-\frac{\alpha_{1}^{2} / 4}{\left(\xi^{2}-\left(i \omega-\frac{(\mathbf{P Q})}{2 m}-\lambda\right)^{2}\right)^{2}}\right)\right] \\
& +\frac{\alpha_{1}^{3}}{4} \cos \left(\frac{(\mathbf{Q r})}{\hbar}\right)\left[\frac { 1 } { ( - i \omega + \xi - \frac { 3 ( \mathbf { P Q } ) } { 2 m } + \lambda ) ( i \omega + \xi - \frac { ( \mathbf { P Q } ) } { 2 m } - \lambda ) } \left(\frac{1}{\xi^{2}-\left(i \omega-\frac{(\mathbf{P Q})}{2 m}-\lambda\right)^{2}}\right.\right. \\
& \left.-\frac{\alpha_{1}^{2} / 4}{\left(\xi^{2}-\left(i \omega-\frac{(\mathbf{P Q})}{2 m}-\lambda\right)^{2}\right)^{2}}\right)+\frac{1}{\left(-i \omega+\xi+\frac{3(\mathbf{P Q})}{2 m}+\lambda\right)\left(i \omega+\xi+\frac{(\mathbf{P Q})}{2 m}-\lambda\right)} \\
& \left.\left.\cdot\left(\frac{1}{\xi^{2}-\left(i \omega+\frac{(\mathbf{P Q})}{2 m}-\lambda\right)^{2}}-\frac{\alpha_{1}^{2} / 4}{\left(\xi^{2}-\left(i \omega+\frac{(\mathbf{P Q})}{2 m}-\lambda\right)^{2}\right)^{2}}\right)\right]\right\}
\end{aligned}
$$




$$
\begin{aligned}
& F_{2}\left(\mathbf{r}, \mathbf{r}, \tau=\tau^{\prime}\right)=T \sum_{\omega} \chi_{0}(z) \chi_{0}^{*}(z) \int_{-\infty}^{\infty} \frac{m d \xi}{(2 \pi \hbar)^{2}} \int_{0}^{2 \pi} d \varphi \frac{\alpha_{1}^{5}}{32}\left\{\cos \left(\frac{(\mathbf{Q r})}{\hbar}\right)\right. \\
& {\left[4 \cdot \left(\frac{1}{\left(i \omega+\xi-\frac{(\mathbf{P Q})}{2 m}-\lambda\right)^{3}\left(-i \omega+\xi+\frac{(\mathbf{P Q})}{2 m}+\lambda\right)^{2}\left(-i \omega+\xi-\frac{3(\mathbf{P Q})}{2 m}+\lambda\right)}\right.\right.} \\
& \left.+\frac{1}{\left(i \omega+\xi+\frac{(\mathbf{P Q})}{2 m}-\lambda\right)^{3}\left(-i \omega+\xi-\frac{(\mathbf{P Q})}{2 m}+\lambda\right)^{2}\left(-i \omega+\xi+\frac{3(\mathbf{P Q})}{2 m}+\lambda\right)}\right) \\
& +3\left(\frac{1}{\xi^{2}-\left(i \omega-\frac{3(\mathbf{P Q})}{2 m}-\lambda\right)^{2}\left(\xi^{2}-\left(i \omega+\frac{(\mathbf{P Q})}{2 m}-\lambda\right)^{2}\right)^{2}}\right. \\
& \left.\left.+\frac{1}{\left(\xi^{2}-\left(i \omega+\frac{3(\mathbf{P Q})}{2 m}-\lambda\right)^{2}\right)\left(\xi^{2}-\left(i \omega-\frac{(\mathbf{P Q})}{2 m}-\lambda\right)^{2}\right)^{2}}\right)\right]+2 \cos \left(\frac{3(\mathbf{Q r})}{\hbar}\right) \\
& \cdot\left[\left(\frac{1}{\left(i \omega+\xi+\frac{5(\mathbf{P Q})}{2 m}-\lambda\right)\left(\xi^{2}-\left(i \omega-\frac{3(\mathbf{P Q})}{2 m}-\lambda\right)^{2}\right)\left(-i \omega+\xi+\frac{3(\mathbf{P Q})}{2 m}+\lambda\right)\left(\xi^{2}-\left(i \omega+\frac{(\mathbf{P Q})}{2 m}-\lambda\right)^{2}\right)}\right.\right. \\
& \left.+\frac{1}{\left(i \omega+\xi-\frac{5(\mathbf{P Q})}{2 m}-\lambda\right)\left(\xi^{2}-\left(i \omega+\frac{3(\mathbf{P Q})}{2 m}-\lambda\right)^{2}\right)\left(-i \omega+\xi-\frac{3(\mathbf{P Q})}{2 m}+\lambda\right)\left(\xi^{2}-\left(i \omega-\frac{(\mathbf{P Q})}{2 m}-\lambda\right)^{2}\right)}\right) \\
& +\left(\frac{1}{\left(\xi^{2}-\left(i \omega-\frac{3(\mathbf{P Q})}{2 m}-\lambda\right)^{2}\right)\left(\xi^{2}-\left(i \omega+\frac{(\mathbf{P Q})}{2 m}-\lambda\right)^{2}\right)\left(-i \omega+\xi+\frac{3(\mathbf{P Q})}{2 m}+\lambda\right)\left(i \omega+\xi+\frac{(\mathbf{P Q})}{2 m}-\lambda\right)}\right. \\
& \left.\left.+\frac{1}{\left(\xi^{2}-\left(i \omega+\frac{3(\mathbf{P Q})}{2 m}-\lambda\right)^{2}\right)\left(\xi^{2}-\left(i \omega-\frac{(\mathbf{P Q})}{2 m}-\lambda\right)^{2}\right)\left(-i \omega+\xi-\frac{3(\mathbf{P Q})}{2 m}+\lambda\right)\left(i \omega+\xi-\frac{(\mathbf{P Q})}{2 m}-\lambda\right)}\right)\right] \\
& +\cos \left(\frac{5(\mathbf{Q r})}{\hbar}\right)\left(\frac{1}{\left(\xi^{2}-\left(i \omega-\frac{5(\mathbf{P Q})}{2 m}-\lambda\right)^{2}\right)\left(\xi^{2}-\left(i \omega+\frac{3(\mathbf{P Q})}{2 m}-\lambda\right)^{2}\right)\left(\xi^{2}-\left(i \omega-\frac{(\mathbf{P Q})}{2 m}-\lambda\right)^{2}\right)}\right. \\
& \left.\left.+\frac{1}{\left(\xi^{2}-\left(i \omega+\frac{5(\mathbf{P Q})}{2 m}-\lambda\right)^{2}\right)\left(\xi^{2}-\left(i \omega-\frac{3(\mathbf{P Q})}{2 m}-\lambda\right)^{2}\right)\left(\xi^{2}-\left(i \omega+\frac{(\mathbf{P Q})}{2 m}-\lambda\right)^{2}\right)}\right)\right\}
\end{aligned}
$$




$$
\begin{aligned}
& F_{3}\left(\mathbf{r}, \mathbf{r}, \tau=\tau^{\prime}\right)=-T \sum_{\omega} \chi_{0}(z) \chi_{0}^{*}(z) \int_{-\infty}^{\infty} \frac{m d \xi}{(2 \pi \hbar)^{2}} \int_{0}^{2 \pi} d \varphi \frac{\alpha_{1}^{2} \alpha_{2}}{8}\left\{3 \cos \left(\frac{(\mathbf{Q r})}{\hbar}\right)\right. \\
& \cdot\left(\frac{1}{\left(\xi^{2}-\left(i \omega+\frac{(\mathbf{P Q})}{2 m}-\lambda\right)^{2}\right)\left(\xi^{2}-\left(i \omega-\frac{3(\mathbf{P Q})}{2 m}-\lambda\right)^{2}\right)}+\frac{1}{\left(\xi^{2}-\left(i \omega-\frac{(\mathbf{P Q})}{2 m}-\lambda\right)^{2}\right)\left(\xi^{2}-\left(i \omega+\frac{3(\mathbf{P Q})}{2 m}-\lambda\right)^{2}\right)}\right) \\
& +2 \cos \left(\frac{3(\mathbf{Q r})}{\hbar}\right)\left[\frac { 1 } { \xi ^ { 2 } - ( i \omega - \frac { 3 ( \mathbf { P Q } ) } { 2 m } - \lambda ) ^ { 2 } } \cdot \left(\frac{1}{\left(i \omega+\xi+\frac{5(\mathbf{P Q})}{2 m}-\lambda\right)\left(-i \omega+\xi-\frac{(\mathbf{P Q})}{2 m}+\lambda\right)}\right.\right. \\
& \left.+\frac{1}{\left(i \omega+\xi-\frac{3(\mathbf{P Q})}{2 m}-\lambda\right)\left(-i \omega+\xi-\frac{(\mathbf{P Q})}{2 m}+\lambda\right)}+\frac{1}{\left(i \omega+\xi-\frac{3(\mathbf{P Q})}{2 m}-\lambda\right)\left(-i \omega+\xi-\frac{5(\mathbf{P Q})}{2 m}+\lambda\right)}\right) \\
& +\frac{1}{\xi^{2}-\left(i \omega+\frac{3(\mathbf{P Q})}{2 m}-\lambda\right)^{2}} \cdot\left(\frac{1}{\left(i \omega+\xi-\frac{5(\mathbf{P Q})}{2 m}-\lambda\right)\left(-i \omega+\xi+\frac{(\mathbf{P Q})}{2 m}+\lambda\right)}\right. \\
& \left.\left.+\frac{1}{\left(i \omega+\xi+\frac{3(\mathbf{P Q})}{2 m}-\lambda\right)\left(-i \omega+\xi+\frac{(\mathbf{P Q})}{2 m}+\lambda\right)}+\frac{1}{\left(i \omega+\xi+\frac{3(\mathbf{P Q})}{2 m}-\lambda\right)\left(-i \omega+\xi+\frac{5(\mathbf{P Q})}{2 m}+\lambda\right)}\right)\right]+\cos \left(\frac{5(\mathbf{Q r})}{\hbar}\right) \\
& {\left[\left(\frac{1}{\left(\xi^{2}-\left(i \omega-\frac{5(\mathbf{P Q})}{2 m}-\lambda\right)^{2}\right)\left(\xi^{2}-\left(i \omega+\frac{3(\mathbf{P Q})}{2 m}-\lambda\right)^{2}\right)}+\frac{1}{\left(\xi^{2}-\left(i \omega+\frac{5(\mathbf{P Q})}{2 m}-\lambda\right)^{2}\right)\left(\xi^{2}-\left(i \omega-\frac{3(\mathbf{P Q})}{2 m}-\lambda\right)^{2}\right)}\right)\right.} \\
& +2\left(\frac{1}{\left(\xi^{2}-\left(i \omega-\frac{5(\mathbf{P Q})}{2 m}-\lambda\right)^{2}\right)\left(-i \omega+\xi-\frac{3(\mathbf{P Q})}{2 m}+\lambda\right)\left(i \omega+\xi-\frac{(\mathbf{P Q})}{2 m}-\lambda\right)}\right. \\
& \left.\left.\left.+\frac{1}{\left(\xi^{2}-\left(i \omega+\frac{5(\mathbf{P Q})}{2 m}-\lambda\right)^{2}\right)\left(-i \omega+\xi+\frac{3(\mathbf{P Q})}{2 m}+\lambda\right)\left(i \omega+\xi+\frac{(\mathbf{P Q})}{2 m}-\lambda\right)}\right)\right]\right\}
\end{aligned}
$$

\section{References}

1. Zhang, T., et al.: Nat. Phys. 6, 104 (2010)

2. Ge, J.-F., et al.: Nat. Mater. 14, 285 (2015)

3. Ovchinnikov, Yu. N..: Int. J. Modern Phys. B 30(N25), 1650183(1-10) (2016)

4. Ovchinnikov, Yu. N..: JETP 123, 838 (2016)
5. Landau, L.D., Lifshitz, E.M.: Quantum Mechanics. Pergamon, New York (1965)

6. Rashba, E.I.: Sov. Phys. Solid 2, 1109 (1960)

7. Abrikosov, A.A., Gorkov, L.P., Dzyaloshinskii, I.E.: Quantum Field Theory in Statist. Physics. Prentice Hall Inc., Englewood Cliffs (1963)

8. Zwicknagl, G., Jahns, S., Fulde, P.: J. Phys. Soc. Jpn. 86, 083701 (2017) 